\title{
Phenomenological description of seismic sources in a pre-stressed, self-gravitating, thermo-visco-elastic earth model
}

\author{
M. Bonafede $(*)-$ E. Boschi $(* *)$ \\ Received on September 26 th, 1977.
}

Riassunto - Le equazioni del moto per un modello di terra self-gravitante, termo-clastico, soggetto a pre-sforzo, in presenza di una dislocazione, sono risolte formalmente tramite uno sviluppo del campo di spostamento in oscillazioni proprie. Si mostra come la non-hermiticità dell'operatore di convoluzione che appare nelle equazioni, non consente di includere gli effetti visco-elastici nel modello matematico dello stress. Conseguentemente il tensore momento di «stress glut » non può essere considerato rigorosamente localizzato entro la sorgente sismica.

SUMMARY - The equations of motion for a pre-stressed, self-gravitating, thermo-viscolastic Earth model undergoing a dislocation are solved through a normal mode expansion of the displacement field. The non-hermiticity of the involved convolution operator is shown not to allow the stress relaxation to be included in the model stress. Accordingly, the stress glut moment tensor cannot be assumed to be strictly localized within the seismic source region.

\section{INTRODUCTION.}

According to the dislocation theory, earthquakes are caused by step-like discontinuities in the displacement field within the Earth, produced by various possible mechanisms, such as tectonic movements related to convection cells, sudden phase changes of bulk materials, etc. When a dislocation takes place in a purely elastic medium, which was previously in a static state, displacement waves are developed which, according to Burridge and Knopoff (1964), may be interpreted

(*) Dipartimento di Scienze della Terra - Università di Ancona.

$\left(^{* *}\right)$ Istituto di Fisica - Università di Bologna. 
as forced oscillations generated by equivalent body forces, localized within the fault region, acting in absence of the dislocation.

An elegant and detailed account of the various possible phenomenological descriptions of seismic sources in terms of equivalent body forces and moment tensors has been recently given by Backus and Mulcahy (1976). According to their paper, the stress drop tensor, introduced by Gilbert (1971), is unsuitable for the description of a seismic source in a realistic Earth model; the "stress glut " tensor, $\Gamma_{i j}$, is introduced in its place, which is defined as a purely mathematical concept given by the difference between a model stress tensor $\sigma_{i j}$ employed for the mathematical description of the medium, and the true physical stress $\tau_{i j}$. If it is possib'e to introduce in $\sigma_{i j}$ all the stress contributions to the real stress acting outside the physical source region, then $\Gamma_{i j}$ is found to possess the noteworthy property of being localized within the source region $\Sigma$ (i. e. of being zero elsewhere).

The application of these considerations was proved to be successful for the pre-stressed, self-gravitating, non-rotating, elastic Earth model given by Dahlen (1972).

The stress glut yields a unique description of the source and viceversa; however the motion produced by the exciting source in the Earth only determines the equivalent body forces, so that additional information regarding the source region must be employed in order to determine the stress glut.

However, the assumption of a purely elastic medium laying under the conventional dislocation theory, cannot be considered satisfactory for the real Earh, particularly for the longer period oscillatory modes which suffer significant attenuation before completing the first cycle. In view of the increasing interest in the normal modes expansion technique for the determination of earthquake source parameters, we deem it necessary to re-examine the mathematical description of seismic sources in order to account explicitely for the imperfections in the elastic behaviour of the Earth thrcugh the inclusion in the field equations of thermal and visco-elastic effects, which must be present in any realistic Earth model, particularly in presence of large stress variations which develop around fault surfaces and at high temperatures, which, apart from being raised at the fault surface by frictional processes, exist wherever in the deep Earth interior. The perturbative method of solution employed here will show that the purely elastic normal modes can be considered as zero order approximations to the real modes and that 
dispersive and dissipative terms are present in the higher order approximate solutions; such terms can be explicitely derived from eq. [39] once the visco-elastic behaviour of the Earth is known.

Furthermore when viscoelastic effects are considered, then it is found that some restrictions are to be imposed to the form of the model stress tensor, and hence, to the stress glut in order to be able to exploit a normal mode expansion for the solutions of the equations of motion. These restrictions furthermore do not allow he resulting stress glut to be localized within the source region.

\section{BASIC EQLATIONS.}

The equations of motion for a pre-stressed, self-gravitating, thermo viscoelastic medium have not been derived yet, as far as we know, but may be easily written down for a body which was initially at rest and is let into motion at time $t_{0}$ by some excitation mechanism. If $T_{i j}{ }^{(0)}$ is employed to denote the static pre-stress field at a point $\mathbf{x}$ we may write (see, e. g., Jaunzemis, 1977):

$$
\begin{gathered}
T^{(0)}{ }_{i j, i}(\mathbf{x})-\rho^{(0)}(\mathbf{x}) \psi_{, i}{ }^{(0)}(\mathbf{x})=0 \\
\text { when } t<t_{0} \\
\psi_{, u^{(0)}}=4 \pi K \rho^{(0)}
\end{gathered}
$$

where the summation convention over repeated indexes is employed, $\rho^{(0)}$ is the mass density, $\psi^{(0)}$ the gravitational potential, $K$ the gravitational constant, and a comma preceding a subscipt denotes partial differentiation with respect to spatial coordinates.

At a subsequent time $t>t_{0}$, after a dislocation has taken place, a displacement field $s_{l}(\mathbf{x}, t)$ is generated and the dynamic equations of motion, written down for the same material particles read

$$
T_{i, j}(\mathrm{x}+\mathrm{s}, t)-\rho(\mathrm{x}+\mathrm{s}, t) \psi_{, i}(\mathbf{x}+\mathbf{s}, t)=\rho \partial_{t}{ }^{2} s_{i}
$$

while Poisson equation is

$$
\psi_{, i i}=4 \pi k \rho
$$


Writing the dynamic fields in terms of additive perturbations, designed by superscript (1), we have

$$
\begin{gathered}
T_{i j}(\mathbf{x}, t)=T_{i j}^{(0)}(\mathbf{x})+T_{i j}^{(1)}(\mathbf{x}, t) \\
\psi(\mathbf{x}, t)=\psi^{(0)}(\mathbf{x})+\psi^{(1)}(\mathbf{x}, t) \\
\rho(\mathbf{x}, t)=\rho^{(0)}(\mathbf{x})+\rho^{(1)}(\mathbf{x}, t)
\end{gathered}
$$

Assuming, as usual, (see e. g. Dahlen, 1975) $s_{i}$ to be small with respect to the typical scale length of spatial variation of physical properties within the Earth, $T_{i j}{ }^{(0)}, T_{i j}{ }^{(1)}, \psi^{(0)}, \psi^{(1)}$ may be expanded to first order in $s$ at $t>t_{0}$. According to the usual linearization procedure, the convective terms $(\mathbf{s} \cdot \nabla) \mathbf{s}$ are neglected in the r. h. s. of eq. [3] and [1]. Subtraction of eq. [1] from [j], together with [5-7] then yields, to first order in $s$

$$
\tau_{i j, j}-\rho^{(1)} \psi_{, i}{ }^{(0)}-\rho^{(0)} \psi_{, i}{ }^{(1)}=\rho^{(0)} \partial_{l}{ }^{2} s_{i}
$$

where the incremental stress tensor $\tau_{i j}$ is given by

$$
\tau_{i j}(\mathbf{x}, t)=T_{i j}^{(1)}(\mathbf{x}, t)+s_{k} T^{(0)}{ }_{i, k}(\mathbf{x}) .
$$

In the same way we get, from eq. [2], [4], [6] and the mass continuity equation

$$
\begin{gathered}
\psi_{, i i}^{(1)}=4 \pi k \rho^{(1)} \\
\rho^{(1)}=-\left(\rho^{(0)} s_{k}\right)_{, k} .
\end{gathered}
$$

Both terms in the r. h. s. of eq. [9] undergo viscous relaxation while time progresses.

The rotation of the Earth would complicate the form of the previous equation, mainly through the appearence of the Coriolis acceleration. The influence of rotation on the normal modes of an elastic Earth model has been recently discussed by Dahlen \& Smith (1975). Apart from the lowest eigenfrequencies, the perturbation produced by the Cariolis term, consists of a small shift in the frequencies and splitting of the spectral lines in sets of degenerate multiplets. More important for our concern, however, is the non-orthogonality of the normal modes in the rotating case. We shall neglect the rotation of the Earth, specifically in order to avoid this additional complication.

Thermodynamic quantities are governed by he enropy equation 


$$
T_{0} \partial_{t} \eta=q_{i, i}+r
$$

where $T_{0}$ is the constant absolute temperature of the reference state, (i. e. the state at $t<t_{0}$ ), $\eta$ is the entropy per unit volume measured from the value $\eta_{0}$ of the reference state, $q_{i}$ are the components of the heat flux vector and $r$ is the heat supplied per unit volume and unit time.

When the incremental stress tensor $\tau_{i j}$ is described starting from the initial static configuration as reference configuration, it is given by the unsymmetric Piola-Kirchoff tensor (Dahlen, 1975). We can then write the constitutive relation for a thermo-visco-elastic pre-stressed body (see e. g. Fung, 1965)

$$
\tau_{i j}(\mathbf{x}, t)=\int_{t_{0}}^{t} g_{i j k l}(\mathbf{x}, t-\tau) \frac{\partial s_{k l}}{\partial \tau}(\mathbf{x}, \tau) d \tau-\beta_{i j}(\mathbf{x}) \theta(\mathbf{x}, t)
$$

where $\beta_{i j}$ are the thermo-elastic coefficients, $0=T-T_{0}$ is the incremental temperature above $T_{0}$, and the 4-th order tensor $g_{i j k l}$ under the convolution integral is assumed to enjoy only the major symmetry property in the exchange of the first couple wih the second couple of indexes, as is the case of simple elastic pre-stressed media, which may be considered as the limiting case given by eq. [13] when $t \rightarrow t_{0}{ }^{+}$.

Further equations give the constitutive relations for the thermodynamic field:

$$
\begin{gathered}
q_{i}=-k_{i j} T_{, j} \\
\eta=\beta_{i j} S_{i, j}+\frac{c_{n}}{I_{0}} \theta
\end{gathered}
$$

where $k_{i j}$ are the heat transfer coefficients, $T$ is the absolute temperature and $c_{r}$ is the specific heat at constant volume.

SEISMIC SOURCE DESCRIPTION.

Boschi (1973) showed that a dislocation taking place in a thermovisco-elastic medium, produces a displacement field and a temperature field which may be interpreted as resulting from the mechanical 
excitation given by equivalent body forces $\gamma_{1}{ }^{2}$ and the thermal excitation by equivalent heat sources $r^{-}$localized at the fault surface, acting in absence of the dislocation. Thus the equations [8] and [12] may be written, after the dislocation has taken place, in the form:

$$
\begin{gathered}
\sigma_{i, i}-\rho^{(1)} \psi_{, i}^{(0)}-\rho^{(0)} \psi_{, i}^{(1)}=\rho^{(0)} \partial_{t}^{2} s_{i}-\rho^{(0)} \gamma_{i}{ }^{-} \\
T_{0} \partial_{t} \eta=q_{i, j}+r^{-}
\end{gathered}
$$

where $\sigma_{i j}=\tau_{i i}$ away from $\Sigma$ and $\gamma_{1}^{\Sigma}=\tau_{i j, j}-\sigma_{i, j i}$. «Static " heat sources $r$ are neglected for consistency sake with the assumption of a static reference configuration. If we intend to obtain a solution of eq. [16] through an expansion of the displacement field in terms of the normal modes for an elastic Earth model, thermal non-adiabatic and viscous decay of stress must be taken apart from the model stress, leaving in $\sigma_{i j}$ only the purely elastic component for adiabatic motions, given by the following relations:

$$
\begin{gathered}
\sigma_{i j}=\sigma_{i j}{ }^{(a d)}-\sigma_{i j}{ }^{(v+r)} \\
\theta=\theta^{(a d)}+\theta^{(r)}
\end{gathered}
$$

where

$$
\begin{gathered}
\theta^{(a d l)}=\frac{T_{0}}{\rho c_{v}} \beta_{k, l} s_{t, l} \\
\sigma_{i j}{ }^{(n d)}=\Lambda_{i j k i} s_{k, l}=g_{i j k l}(\mathbf{x}, 0) s_{k, l}(\mathbf{x}, t)+\frac{T_{0}}{\rho c_{v}} \beta_{i j} \beta_{k l} s_{k, l} \\
\sigma_{i j}{ }^{(p+r)}=\int_{t^{+}}^{t} \frac{\partial}{\partial \tau} g_{i j k l}(\mathbf{x}, t-\tau) s_{k, l}(\tau) d \tau+\beta_{i j} \theta^{(r)}
\end{gathered}
$$

where $\Lambda_{i j k l}$ is implicitely defined in eq. [20] and superscript (ad) marks the adiabatic contribution, while $(v)$ marks the viscous contribution and $(r)$ the contribution of the equivalent heat sources.

The non-adiabatic temperature rise, linked to $r^{2}$ appears as an inhomogeneous term and may thus be included in $\gamma_{1}{ }^{2}$.

Let us now consider the homogenenous equation associated with 
eq. [16]:

$$
\sigma_{i j, j}-\rho^{(1)} \psi_{, i}^{(0)}-\rho^{(0)} \psi_{, i^{(1)}}-\rho^{(0)} \partial_{l}^{2} s_{i}=0 .
$$

Eq. [22] may be written in operatorial notation, once the explicit solution for $\psi^{(1)}$ is written down in terms of the Green function $\frac{1}{|\mathbf{x}-\mathbf{y}|}$ :

$$
\begin{aligned}
Q s_{i}= & \rho^{(0)} \partial_{t}{ }^{2} s_{i}+\psi i^{(0)}\left(\rho^{(0)} s_{k}\right),{ }_{k}+K \rho^{(0)}\left\{\int_{V} \frac{\left(\rho^{(0)}(\mathbf{y}) S_{:}(\mathbf{y})\right)_{, k}}{\mathbf{x}-\mathbf{y} \mid} d V_{y}-\right. \\
& \left.-\int_{\partial V} \rho^{(0)}(\mathbf{y}) \frac{1}{|\mathbf{x}-\mathbf{y}|} s_{k}(\mathbf{y}) n_{k} d S_{y}\right\}-\left(\Lambda_{i j k l} s_{k, l}\right)_{, j}+ \\
& +\left\{\int_{i_{0}}^{l} \frac{\partial}{\partial \tau} g_{i j k l}(\mathbf{x}, t-\tau) s_{k, l}(\tau) d \tau\right\}, j=0 .
\end{aligned}
$$

$Q$ is an integro-differential operator, acting on the spatial and temporal dependence of the displacement field: it may be splitted in the form

$$
Q=\tau^{A}+\Xi^{A}+\tau^{B} \Xi^{B}
$$

where $\tau^{A}$ and $\tau^{B}$ act on the temporal dependence and $\Xi^{A}, \Xi^{B}$ on the spatial dependence of $s(\mathbf{x}, t)$.

Let us consider for the moment the operator

$$
Q^{A}=\tau^{A}+\Xi^{A}
$$

The reduced equation

$$
Q^{A} s_{t}=0
$$

is the usual equation of elastic normal modes and is well-known to posses a complete set of oscillatory solutions, which will be termed « reduced normal modes» and be denotes as

$$
\mathbf{s}^{(\alpha)}=\mathbf{u}^{(\alpha)}(\mathbf{x}) \exp \left(i \omega_{\alpha} t\right) .
$$

Since $Q^{(A)}$, under appropriate boundary conditions (Dahlen, 1975), is a hermitian operator, the reduced normal modes may be assumed to 
constitute a complete and orthonormal set in $V$ if the measure $\rho^{(0)} d V$ is employed

$$
\left\langle\mathbf{u}^{(\alpha)} \cdot \mathbf{u}^{(\beta)}\right\rangle=\int_{i} \rho^{(0)} u_{i}^{(\alpha)} u_{i}^{(\beta)^{*}} d V=\delta_{a \beta}
$$

where an asterisk denotes complex coniugation.

When $t \rightarrow t_{0}{ }^{+}, \tau^{B} \rightarrow 0$, the reduced normal modes may be interpreted as the real modes propagating immediately after the displacement field has been set up by the dislocation. The displacement field $s(\mathbf{x}, t)$ may be expanded in reduced normal medes in the form

$$
s_{i}(\mathbf{x}, t)=\sum_{a} A_{\alpha}(t) u_{i}^{(a)}(\mathbf{x})
$$

After inserting [29] in [16], remembering that $Q^{A} s=0$ and taking the scalar product of the resulting equation with $\mathbf{u}^{(\beta)}$ eq. [16] can be written in the form

$$
\frac{d^{2} A_{\rho}}{d t^{2}}+\omega_{\beta}^{2} A_{\beta}+\sum_{\alpha} \int_{t_{0}}^{\ell} G^{(\alpha, \beta)}(t-\tau) A_{\alpha}(\tau) d \tau=\left\langle\gamma_{i}^{z}, u_{i}^{(\beta)}\right\rangle
$$

where

$$
G^{(\alpha, \beta)}(t-\tau)=\left\langle\frac{1}{\rho^{(0)}} \frac{\partial}{\partial_{i}}-g_{i j k l}(i-\tau) u_{k} l^{(\alpha)} ; u_{i, i}{ }^{(\beta)}\right\rangle
$$

where the boundary condition that $\partial V$ is a free surface both at time $t_{0}{ }^{+}$and at any following time $t$ has been employed.

If the viscoelastic functions $g_{i j k l}$ are real valued, then $G^{(x, \theta)}(t)=$ $=G^{(\beta, \alpha)^{*}}(l)$, i. e. the part of the viscoelastic operator acting on space variables, $\Xi^{R}$, is hermitian.

Consider now the homogeneous equation associated with eq. [30]

$$
\frac{d^{2} A_{\beta}}{d t^{2}}+\omega_{\beta}^{2} A_{\beta}=-\Sigma_{{ }_{1}} \int_{i_{0}}^{\prime} G^{(\alpha, \beta)}(t-\tau) A_{\alpha}(\tau) d \tau
$$

Causality principium requires the kernel $G^{(\alpha, \beta)}(t, \tau)$ to be zero if $\tau>t$ which allows the integral operator 


$$
\mathcal{G}^{(\alpha, \beta)}(t)=\int_{t_{0}^{+}}^{t} G^{(\alpha \beta)}(t-\tau)(\cdot) d \tau
$$

to be written as

$$
\mathcal{G}^{(\alpha, \beta)}(t)=\int_{t_{0}^{+}}^{\infty} G^{(\alpha, \beta)}(t-\tau)(\cdot) d \tau .
$$

Here a dot within brackets denotes a function to be specified. $g^{(\alpha, \beta)}$ however is not a hermitian operator. This furthermore implies that the eigenvalue temporal equation [32] cannot be assumed to possess a complete set of solutions. Indeed the operator $\mathcal{G}$ may be splitted in its hermitian and antihermitian parts $\hat{\pi}$ and $\mathscr{A}$

$$
\mathcal{G}=\Re+i \mathscr{L}
$$

where

$$
\begin{aligned}
& \mathcal{\ell}^{(\alpha, \beta)}=\frac{1}{2} \int_{0}^{+\infty}\left[G^{(\alpha, \beta)}(t-\tau)+G^{(\alpha, \beta)^{*}}(\tau-t)\right](\cdot) d \tau \\
& \mathscr{A} \mathcal{L}^{(\alpha, \beta)}=\frac{1}{2} \int_{0}^{+\infty}\left[G^{(\alpha, \beta)}(t-\tau)-G^{(\alpha, \beta)^{*}}(\tau-t)\right](\cdot) d \tau
\end{aligned}
$$

and a perturbative computation would yield the result that viscoelastic waves are both dispersive and dissipative since their frequencies depend on the wave number and furthermore are complex valued, the latter result explicitely preventing the solutions [29] from being a complete set with respect to the time variable. Eq. [30] or [32] may be solved following the iterative scheme, usually employed for the solution of analogous integral equations

$$
\text { 0-order: } \frac{d^{2} A_{i^{(0)}}^{(0)}}{a i^{2}}+\omega_{\beta}{ }^{2} A_{\beta}^{(0)}=\left\langle\gamma_{i}{ }^{2}, u_{i}\right\rangle
$$

$n$-th order: $\frac{d^{2} A_{\beta}^{(n)}}{a^{\prime} i^{2}}+\omega_{\beta}^{2} A_{\beta}^{(n)}=\left\langle\gamma_{i}^{\Sigma}, u_{i}\right\rangle-\sum_{\alpha} \Theta^{(\alpha, \beta)} A_{a}^{(n-1)}$ 
where the viscoelastic relaxation term is included in the inhomogenecus part of the equation. Eq. [39] is then easily solved for $A_{\beta}^{(n)}$ according to the method of variation of constants, once the $(n-1)$ order solution has been obtained.

\section{Conclusions.}

The completeness requirement for the normal mode expansion leads us to consiler the viscoelastic term as an additional equivalent body force. While terms contained in $\gamma_{i}{ }^{2}$ may all be assumed to become vanishingly small away from the fault surface, the viscoelastic equivalent bcdy force surely may not, since viscous relaxation of stress takes place within the whole Earth. Even the static component of the displacement field, in fact, propagates over long distances (Press, 1965), in particular up to the lower mantle, where high temperatures make the stress relaxation function more effective. Furthermore eq. [39] states that viscous dissipation does not allow waves with a definite frequency to propagate independently of each other, since they interact through the non-diagonal elements $\Theta^{(\alpha, \beta)}$. Thus the stress glut tensor

$$
i_{i l}=o_{1 j}{ }^{(\cdots)}:-\tau_{11}
$$

in the general case of couple thermo-viscoelastic media cannot be assumed localized within the souce region, owing to the non-hermiticity of the convolution integral, that prevents the viscous stress relaxation to be included in the model stress tensor $\sigma_{i j}^{(a d)}$ when applying normal mode expansion techniques to the determination of seismic source parameters.

\section{ACKNOWLEDGEMENTS.}

The authors wish to thank prof. M. Caputo for careful reading of the manuscript and useful comments and suggestions.

Work performed in the framework of the Progetto Finalizzato Geodinamica, Publ. N. 10. 


\section{REFERENCES}

Backus, G. and Mulcahy, M., 1976. - Moment tensors and other phenomenological descriptions of seismic sources - I. Continuous displacements. Geophys. J. R. astr. Soc., 46, pp. 341-361.

Boschi, E., 1973. - A thermoviscoelastic model of the earthquake mechanism. "J. Geophys. Res. ». 78, pp. 7733-7737.

Burridge, R. and KNopoff, L., 1964. - Body force equivalents for seismic dislocations. «Bull. Seism. Soc. Am.», 54, pp. 1875-1888.

DAHLEN, F. A., 1972. - Elastic dislocation theory for a self-gravitating elastic configuration with an initial static stress field, "Geophys. J. R. Astr. Soc. », 28, pp. 357-383.

DaHLEN, F. A. and SMITH, M. L., 1975. - The influence of rotation on the free oscillations of the earth. "Phil. Trans. R. Soc. Lond. ", A279, pp. 585-629.

Eringen, A. C., 1967. - Mechanics of continua. John Wiley and Sons, Inc., New York.

Fung, Y. C., 1965. - Foundation of Solid Mechanics, Prentice-Hall, New Jersey.

Gilbert. J. F., 1971. - Excitation of the normal modes of the earth by earthquake sources. "Geophys. J. R. Astr. Soc. ", 22, pp. 223-226.

Jaunzemis, W., 1977. Continuum Mechanics, McMillan, New York.

Press, F., 1965. - Displacements, strains and tilts at teleseismic distances. "J. Geophys. Res. ", 70, pp. 2395-2412. 